\title{
Ehlers-Danlos syndrome type VIII is clinically heterogeneous disorder associated primarily with periodontal disease, and variable connective tissue features
}

\author{
Eyal Reinstein ${ }^{\star,}$, Celia Dawn DeLozier ${ }^{2}$, Ziv Simon ${ }^{3}$, Serguei Bannykh ${ }^{4}$, David L Rimoin ${ }^{1, *}$ and Cynthia J Curry ${ }^{2}$
}

We would like to dedicate this article to the memory of David Rimoin, MD, PhD, who passed away on May 27, 2012. He was an outstanding geneticist, mentor, and human being.

Ehlers-Danlos syndrome (EDS) type VIII (periodontitis type) is a distinct form of EDS characterized by periodontal disease leading to precocious dental loss and a spectrum of joint and skin manifestations. EDS type VIII is transmitted in an autosomal dominant pattern; however, the mutated gene has not been identified. There are insufficient data on the spectrum of clinical manifestations and natural history of the disorder, and only a limited number of patients and pedigrees with this condition have been reported. We present a four-generation EDS type VIII kindred and show that EDS VIII is clinically variable and although some cases lack the associated skin and joint manifestations, microscopic evidence of collagen disorganization is detectable. We further propose that the diagnosis of EDS type VIII should be considered in familial forms of periodontitis, even when the associated skin and joint manifestations are unconvincing for the diagnosis of a connective tissue disorder. This novel observation highlights the uncertainty of using connective tissue signs in clinical practice to diagnose EDS type VIII.

European Journal of Human Genetics (2013) 21, 233-236; doi:10.1038/ejhg.2012.132; published online 27 June 2012

Keywords: Ehlers-Danlos syndrome type VIII; periodontitis; heritable connective tissue disorder

\section{INTRODUCTION}

Periodontitis encompasses a group of conditions characterized by progressive destruction of connective tissue and tooth-supporting structures including the gingiva, periodontal ligament, and alveolar bone. Multiple lines of evidence support the contribution of genetic factors to periodontitis. Genetic studies have identified several loci for genes that contribute to the condition, mainly in families with monogenic immune deficiency disorders or cyclic neutropenia. ${ }^{1}$ Ehlers-Danlos syndrome (EDS) type VIII (OMIM no. 130080) is a rare, distinct connective tissue disorder characterized by periodontal disease causing premature dental loss. The associated clinical abnormalities include a spectrum of joint and skin manifestations with significant clinical overlap with other EDS subtypes. ${ }^{2}$ The diagnosis of EDS type VIII is usually established in an individual presenting with dental and connective tissue abnormalities in the context of a familial autosomal dominant pattern of inheritance. Here, we present a previously unreported four-generation pedigree with EDS type VIII. We show that the clinical manifestations of EDS type VIII can be primarily periodontal and propose that the diagnosis of EDS type VIII should be considered in familial forms of periodontitis even when there is little clinical (joint and skin findings) basis for considering a diagnosis of a connective tissue disorder.

\section{PATIENTS AND METHODS}

The proposita (IV-2; Figures 1 and 2) is a 7-year-old girl who was born after an uncomplicated pregnancy to a 26-year-old mother and 32-year-old father. She was born at term via vaginal delivery and met all developmental milestones. At age 3, the patient was noted to have early gingival disease and some easy shin bruising and occasional nosebleeds. Prior studies included an echocardiogram at age 4 that was normal with the exception of a small PFO, normal ophthalmological examination, and normal activities of Von Willebrand factor and factor VIII. Physical examination at age 5 revealed a normal body habitus (Figure 1), nondysmorphic facial features, normal palate, uvula and sclera. Extremities showed minimal bruising on the shins. There was mild joint hypermobility (Beighton score 2/9), probably within normal limits for her age. Skin was of normal consistency, with no hyperextensibility, and scar formation was normal. A follow-up examinations at ages 7 and 8 did not show additional relevant findings. Dental examination revealed mixed dentition appropriate to her age. Several metal restorations were present on her primary teeth (Figure 1). Generalized severe gingival edema and erythema were observed; these were associated with abundant bacterial biofilm accumulation, and gingival recession was present on some of her primary and permanent teeth. The gingival soft tissues were of poor quality with minimally attached and keratinized gingivae. Several capillaries were visible in the mucosa, accentuating its thin nature.

The family history is significant for her father; paternal grandfather and paternal great-grandfather all are being affected with this condition (Figure 2). The proposita's father (III-2), age 39, had experienced significant shin bruising

\footnotetext{
${ }^{1}$ Medical Genetics Institute, Cedars-Sinai Medical Center, Los Angeles, CA, USA; ${ }^{2}$ Genetic Medicine Central California, UCSF-Fresno, Fresno, CA, USA; ${ }^{3}$ Private practice limited to periodontics, Beverly Hills, CA, USA; ${ }^{4}$ Department of Pathology, Cedars-Sinai Medical Center, Los Angeles, CA, USA

*Correspondence: Dr E Reinstein, Medical Genetics Institute, Cedars-Sinai Medical Center, 8700 Beverly Boulevard, Los Angeles, CA 90048, USA. Tel: +1 301 423 9904; Fax: +1 310423 2080; E-mail: Eyal.Reinstein@cshs.org

"Deceased

Received 24 January 2012; revised 19 May 2012; accepted 21 May 2012; published online 27 June 2012
} 

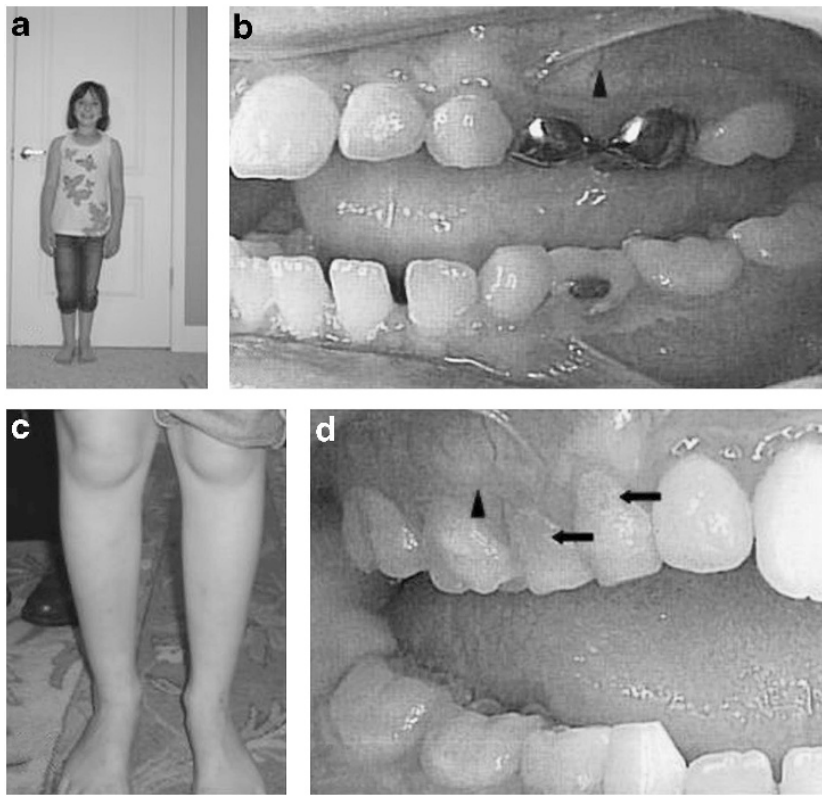

Figure 1 Physical $(\mathbf{a}+\mathbf{c})$ and dental $(\mathbf{b}+\mathbf{d})$ findings in the proband. Note normal body habitus (a), nondysmorphic facial features and minimal shin bruising (c). Several metal restorations are observed on primary teeth (b) as well as gingival recession and bacterial biofilm accumulation (d).

from early teens, resulting in discolored pretibial scarring (Figure 3). $\mathrm{He}$ reported receding gums and periodontal disease and underwent periodontal surgery that included soft tissue grafting at age 20. He started losing teeth in his teens and had lost six permanent teeth at the time of evaluation at age 39. He currently has two loose teeth. Pertinent findings on physical examination included normal body habitus, significant pretibial staining and no joint hypermobility (Beighton score 0/9). The skin was normal without hyperextensibility or abnormal scars. He had no loss of subcutaneous fat and has had no dislocations or other joint symptoms suggestive of a connective tissue disorder. Electron microscopy examination of skin taken from the pretibial region demonstrated decreased collagen content, abnormal variation in collagen fibril diameter and some abnormally shaped fibers suggesting collagen misassembly (Figure 4). No obvious alterations in elastin fibers were observed (data not shown). Dental examination revealed multiple missing teeth and severe gingival inflammation (Figure 3). Soft tissue edema and erythema were mostly notable in the inter-dental areas. Gingival recession was generalized along with multiple areas of poor tissue quality. He was diagnosed with advanced periodontitis (class IV; the most severe form according to the American Dental Association classification). Evidence of previous tissue grafting was visible and was suggesting of palatal donor tissue. Pathological teeth migration was observed and was attributed to the loss of posterior teeth and the advanced attachment loss from periodontal disease. The proposita's grandfather (II-2), deceased at age 62 from colon cancer, had started losing his teeth at age 19 and had full dentures by age 42 . He had severe shin bruising but no joint pain, joint laxity or dislocations by family report. The patient's greatgrandfather (I-1), now age 86, began losing teeth in his early teens. He reported that his teeth became chipped during sleep and that he had mandibular bone loss. By age 21, he had lost 14 permanent teeth; the remaining teeth were extracted while he was a soldier and he has since worn a full set of dentures. He has never had shin bruising, joint laxity, dislocations or joint pain. He recently underwent aortic valve replacement surgery for degenerative calcification and stenosis of the aortic valve. Physical examination revealed a well-healed sternal scar, and again, no joint (Beighton score 0/9) or skin manifestations, suggesting a connective tissue disorder and no discoloration of the shins (Figure 5). Medical history and physical examination in the other family members shown in the pedigree revealed no signs of dental, joint or skin manifestations.

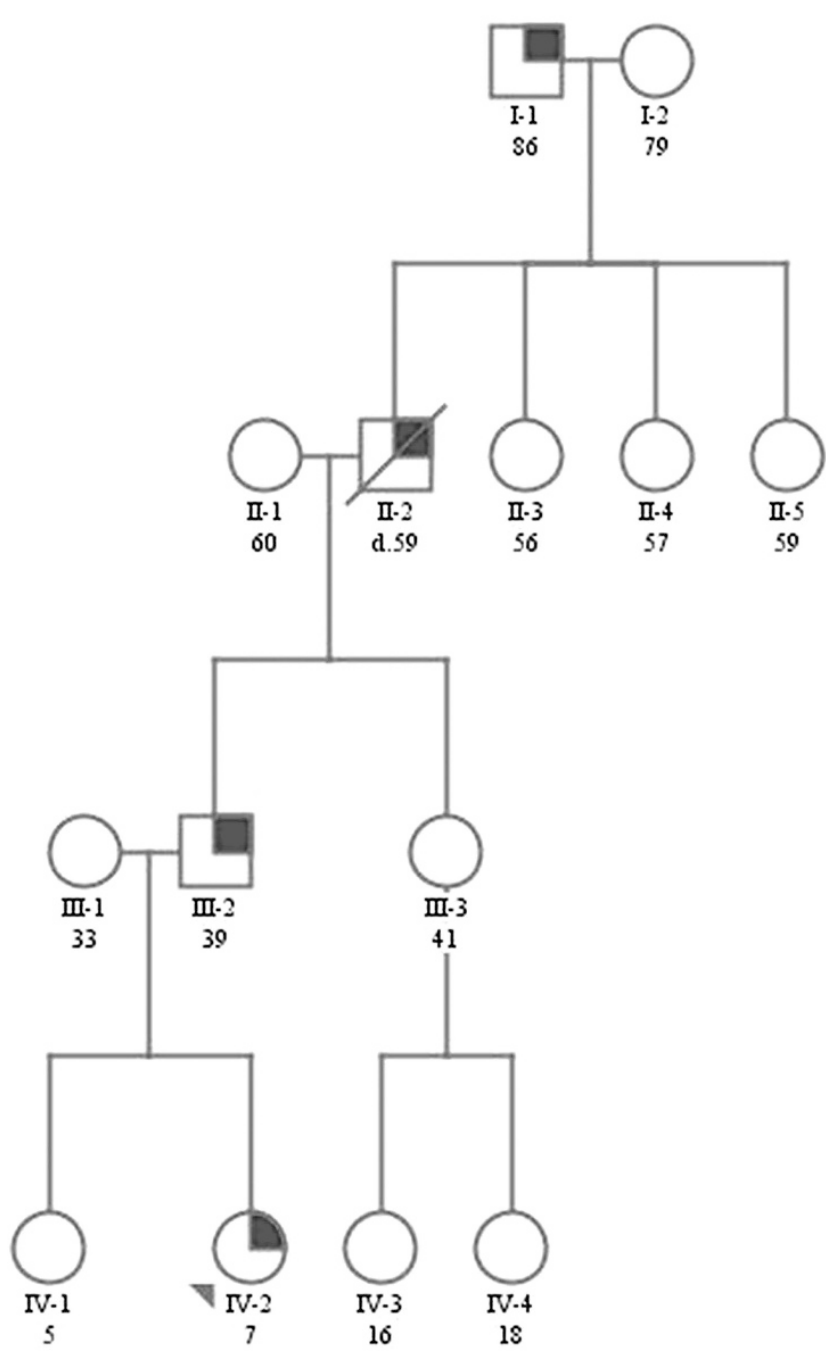

Figure 2 Pedigree of family with EDS type VIII.
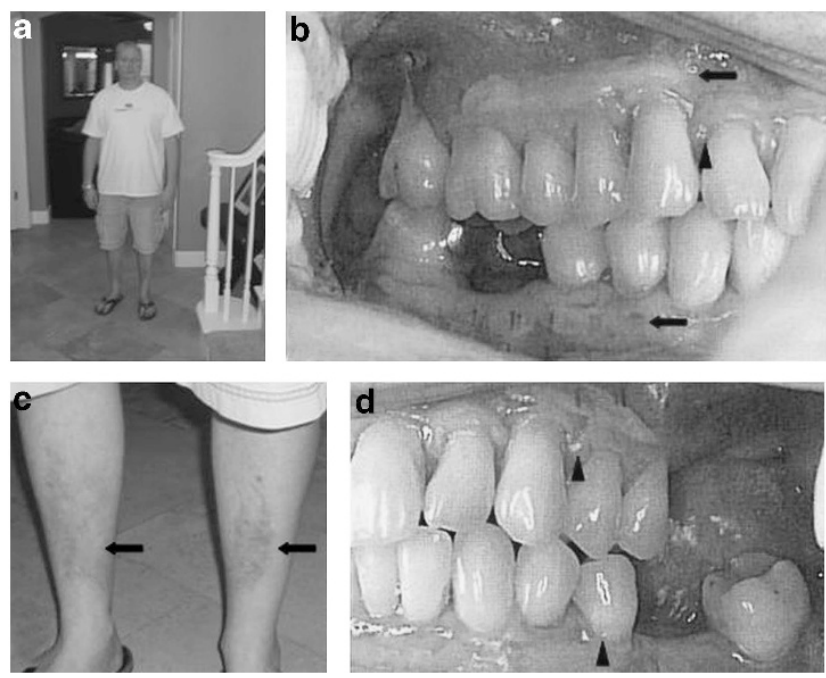

Figure 3 Physical (a, c) and dental (b, d) findings in the proposita's father. Note normal body habitus (a) and pronounced shin discoloration (c). There is generalized periodontal disease with severe recession and multiple missing teeth (b, d). 


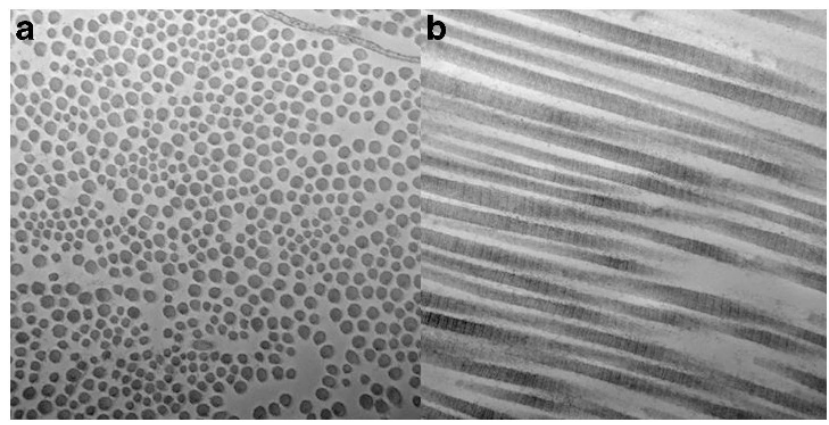

Figure 4 Pretibial dermal collagen morphology in the proposita's father. (a) Cross section demonstrating decreased collagen content, abnormal variation in collagen fibril diameter with some abnormally shaped fibers. (b) Longitudinal section demonstrating normal striation pattern of collagen fibers.

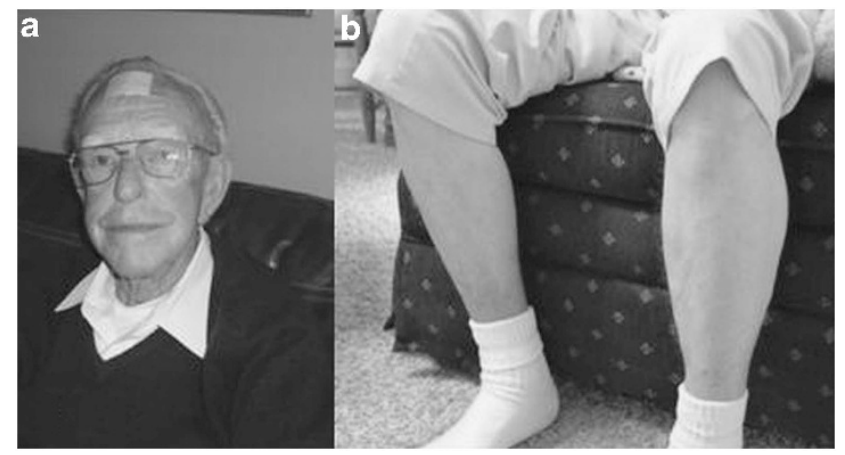

Figure 5 Facial features (a) and shin photo (b) of the proband's greatgrandfather.

\section{DISCUSSION}

This report highlights the variability of connective tissue signs in EDS type VIII, similar to that seen in other forms of EDS.

The EDSs are a diverse group of heritable disorders that are characterized by generalized fragility of connective tissues. The latest (1997) classification recognizes six EDS subtypes, based on clinical characteristics, mode of inheritance and biochemical and molecular findings. A seventh group of 'miscellaneous' forms has been proposed; this group includes EDS type VIII and other rare subtypes. ${ }^{3}$ Over the past few years, several new forms have been clinically and molecularly delineated, including dermatan 4-O-sulfotransferase 1 deficient-EDS, spondylocheiro dysplastic EDS (caused by mutation of the SLC39A13 gene) and the X-linked periventricular heterotopia variant (PH-EDS), caused by mutations in Filamin $A .^{4-7}$

In fact, the existence of EDS type VIII, as a specific EDS form associated with periodontal disease and having a distinct underlying genetic basis, is still debated in the literature. This is based in part on the reported association of gingivitis (and sometimes subsequent periodontitis) with other subtypes of EDS (eg, the classic, hypermobile and vascular forms), ${ }^{3,8}$ arguing that gingival involvement may be considered a nonspecific consequence of various heritable connective tissue disorders. However, based on previously reported cohorts of patients with EDS type VIII, it seems that this is a distinct syndrome with a few major differences from other EDS types. First, the dominant transmission of periodontal disease has very high penetrance, and in previously reported pedigrees as well as the current family, affected individuals show signs of periodontal
Table 1 Clinical features in EDS type VIII

\begin{tabular}{lcc}
\hline Clinical feature & This report & $\begin{array}{c}\text { Previous reports } \\
\text { total/total evaluated }^{\mathrm{a}}\end{array}$ \\
\hline Periodontitis & $4 / 4$ & $54 / 62$ \\
Joint hypermobility & $1 / 4^{\mathrm{b}}$ & $25 / 62$ \\
Joint dislocation & $0 / 4$ & $27 / 62$ \\
Skin hyperextensibility & $0 / 4$ & $27 / 62$ \\
Atrophic scars & $0 / 4$ & $47 / 62$ \\
Pretibial staining & $3 / 4$ & $35 / 62$ \\
EDS IV-like facial features & $0 / 4$ & $19 / 62$ \\
\hline
\end{tabular}

Abbreviation: EDS, Ehlers-Danlos syndrome.

aThis is an approximation. The clinical manifestations of this syndrome are poorly defined as the detailed descriptions of some reported cases are not available. Some features should not be considered as not present but rather as not sought after or not obvious on physical examination.

bMild, probably within normal limits for patient age.

disease, although the skin and joint findings vary (Table 1). Second, periodontal disease is generalized and is usually more severe than the gingivitis encountered in the general population and the other EDS forms. Third, periodontal disease in EDS VIII can affect young children (and deciduous teeth), who in the general population rarely have periodontal disease even in the presence of poor oral hygiene.

Based on previous reports, there is considerable phenotypic heterogenetity in EDS type VIII, in spite of the fact that most patients have presented with significant connective tissue abnormalities. ${ }^{9-23}$ The spectrum of skin manifestations includes skin hyperextensibility, easy bruising, slow wound healing, dystrophic scar formation, pretibial discoloration and abnormal skin histopathology. Several skeletal phenotypes have been associated with EDS type VIII. These include joint laxity and dislocations, Marfanoid habitus, osteopenia, early-onset osteoarthritis, pectus deformity, scoliosis and pes planus. ${ }^{24}$ Recurrent shin bruising resulting in pretibial discoloration has also been described in a few patients with EDS type VIII; histological examination of these lesions revealed nonspecific deposition of hemosiderin consistent with chronic trauma. ${ }^{25}$ Shin bruising was documented in three members of this family, which is the result of an increased skin and small blood vessel fragility, as none of them had excessive trauma that caused their shins to be bruised. The key connective tissue abnormalities in this family as compared with previous reported patients are summarized in Table 1.

The differential diagnosis of autosomal dominant periodontitis includes several other monogenetic syndromes that consistently have significant periodontitis as part of the clinical phenotype. These include neutrophil disorders (eg, cyclic neutropenia, leukocyte adhesion deficiency, etc), immune/skin disorders (eg, Kindler, PapillonLefev̀re and Haim-Munk syndromes) and prepubertal aggressive periodontitis. ${ }^{1}$ However, in most of these syndromes the periodontitis is an associated feature, while the primary clinical manifestations are related to immune dysfunction and/or skin presentation. In addition, most of these conditions are inherited in an autosomal recessive manner. Because of the minimal connective tissue abnormalities observed in the family reported here, some consideration was given to a diagnosis of localized aggressive periodontitis (LAP). LAP is a clinical and genetic heterogeneous group of periodontal disorders that can present with early periodontal disease accompanied by variable degree of neutrophil function abnormalities. Yet, the severe shin bruising observed in some members of this family is not seen in LAP and we have excluded chromosome 1q25, previously linked to $\mathrm{LAP}^{26}$ as a potential causal 
chromosomal region in this family (unpublished results). It has to be mentioned in addition, that one cannot exclude that the coexistence of pretibial staining with periodontal disease observed in this family is a variant of EDS type VIII or alternatively, a separate disorder. Pretibial fragility and staining have been observed in Epidermolysis bullosa, and Bethlem Myopathy, however, this unusual dominantly inherited periodontal disease is not seen in those disorders.

The molecular basis of EDS type VIII remains unknown. In 2003, a linkage study in a five-generation family with EDS type VIII established linkage to chromosome $12 \mathrm{p} 13$ with a LOD score of 5.17. However, that study was unable to identify any pathogenic mutations within the region identified. ${ }^{27}$ We recently completed a genome-wide linkage scan in a different EDS VIII pedigree with a resulting linked region in chromosome 9. As yet, no gene mutation has been identified that could explain the phenotype. ${ }^{28}$ We hope that our ongoing studies will lead to the identification of the diseasecausing gene in EDS type VIII.

\section{CONCLUSIONS}

We suggest that, in contrast to other subtypes of EDS, the periodontal features, rather than the connective tissue abnormalities, serve as the main clinical finding to define EDS type VIII. We propose that the diagnosis of EDS type VIII should be considered in patients presenting with dominant familial forms of early-onset periodontal disease and dental loss even when there is little other clinical basis for considering a connective tissue disorder. Further detailed clinical and gene analysis studies in larger cohorts of EDS type VIII patients are currently underway. These are needed to better delineate the phenotypic spectrum of the disorder; gene identification will allow the development of diagnostic guidelines for EDS VIII that are based also on molecular testing.

\section{CONFLICT OF INTEREST}

The authors declare no conflict of interest.

\section{ACKNOWLEDGEMENTS}

Dr Rimoin appreciated the support from the Steven Spielberg Pediatric Research Center, the NIH/NICHD Program Project Grant (HD36657), the Medical Genetics NIH/NIGMS Training Program Grant (5-T32-GM08243) and the Cedars-Sinai General Clinical Research Center Grant (M01-RR00425) for samples collected under CSMC IRB Protocols 0463 and 4232. We thank the family members for their willing participation in this study and Dr Dee Nishimine for providing dental photos of the patients. We also thank Cecelia Moreno MD and Dwain Wright MD for the referral of this family.

1 Hart TC, Atkinson JC: Mendelian forms of periodontitis. Periodontol 2000 2007; 45: 95-112.

2 Karrer S, Landthaler M, Schmalz G: Ehlers-Danlos type VIII. review of the literature. Clin Oral Investig 2000; 4: 66-69.

3 Beighton P, De Paepe A, Steinmann B, Tsipouras P, Wenstrup RJ: Ehlers-Danlos syndromes: revised nosology, Villefranche, 1997. Ehlers-Danlos National Foundation
(USA) and Ehlers-Danlos Support Group (UK). Am J Med Genet 1998; 77 31-37.

4 Shimizu K, Okamoto N, Miyake N et al: Delineation of dermatan 4-O-sulfotransferase 1 deficient Ehlers-Danlos syndrome: observation of two additional patients and comprehensive review of 20 reported patients. Am J Med Genet A 2011; 155A: 1949-1958.

5 Gómez-Garre P, Seijo M, Gutiérrez-Delicado E et al: Ehlers Danlos syndrome and periventricular nodular heterotopia in a Spanish family with a single FLNA mutation. J Med Genet 2006; 43: 232-237.

6 Sheen VL, Jansen A, Chen MH et al: Filamin A mutations cause periventricular heterotopia with Ehlers-Danlos syndrome. Neurology 2005; 64: 254-262.

7 Giunta C, Elçioglu NH, Albrecht B et al: Spondylocheiro dysplastic form of the Ehlers-Danlos syndrome - an autosomal-recessive entity caused by mutations in the zinc transporter gene SLC39A13. Am J Hum Genet 2008; 82: 1290-1305.

8 Castori M, Camerota F, Celletti $\mathrm{C}$ et al: Natural history and manifestations of the hypermobility type Ehlers-Danlos syndrome: a pilot study on 21 patients. Am J Med Genet A 2010; 152: 556-564.

9 Stewart RE, Hollister DW, Rimoin DL: A new variant of Ehlers-Danlos syndrome: an autosomal dominant disorder of fragile skin, abnormal scarring, and generalized periodontitis. Birth Defects 1977 XIII: 85-93.

10 Linch $\mathrm{DC}$, Acton $\mathrm{CH}$ : Ehlers-Danlos syndrome presenting with juvenile destructive periodontitis. Br Dent J 1979; 147: 95-96.

11 Cunniff C, Williamson-Kruse L: Ehlers-Danlos syndrome, type viii presenting with periodontitis and prolonged bleeding time. Clin Dysmorphol 1995; 4 145-149.

12 Nelson DL, King RA: Ehlers-Danlos syndrome type VIII. J Am Acad Dermatol 1981; 5: 297-303.

13 Spranger S, Spranger M, Kirchhof K, Steinmann B: Ehlers-Danlos syndrome type VIII and leukodystrophy. Am J Med Genet 1996; 66: 239-240.

14 Hernández A, Aguirre-Negrete MG, Ramírez-Soltero S et al: A distinct variant of the Ehlers-Danlos syndrome. Clin Genet 1979; 16: 335-339.

15 Perez LA, AI-Shammari KF, Giannobile WV, Wang HL: Treatment of periodontal disease in a patient with Ehlers-Danlos syndrome. A case report and literature review. $J$ Periodontol 2002; 73: 564-570.

16 Linch $\mathrm{DC}$, Acton $\mathrm{CH}$ : Ehlers-Danlos syndrome presenting with juvenile destructive periodontitis. Br Dent J 1979; 147: 95-96.

17 Hoffman GS, Filie JD, Schumacher Jr HR et al: Intractable vasculitis, resorptive osteolysis, and immunity to type I collagen in type VIII Ehlers-Danlos syndrome. Arthritis Rheum 1991; 34: 1466-1475.

18 Bond PJ, Friend GW, Meridith MW: Ehlers-Danlos syndrome identified from periodontal findings: case report. Pediatr Dent 1993; 15: 212-213.

19 Moore MM, Votava JM, Orlow SJ, Schaffer JV: Ehlers-Danlos syndrome type VIII: periodontitis, easy bruising, marfanoid habitus, and distinctive facies. J Am Acad Dermatol 2006; 55: S41-S45.

20 Mataix J, Bañuls J, Muñoz C, Bermejo A, Climent JM: Periodontal Ehlers-Danlos syndrome associated with type III and I collagen deficiencies. Br J Dermatol 2008, 158: 825-830.

21 Biesecker LG, Erickson RP, Glover TW, Bonadio J: Molecular and cytologic studies of Ehlers-Danlos syndrome type VIII. Am J Med Genet 1991; 41: 284-288.

22 Dyne KM, Vitellaro-Zuccarello L, Bacchella L, Lanzi G, Cetta G: Ehlers-Danlos syndrome type VIII: biochemical, stereological and immunocytochemical studies on dermis from a child with clinical signs of ehlers-danlos syndrome and a family history of premature loss of permanent teeth. Br J Dermatol 1993; 128: 458-463.

23 Hartsfield Jr JK, Kousseff BG: Phenotypic overlap of Ehlers-Danlos syndrome types IV and VIII. Am J Med Genet 1990; 37: 465-470.

24 Reinstein E, Pariani M, Lachman RS, Nemec S, Rimoin DL: Early-onset osteoarthritis in Ehlers-Danlos syndrome type VIII. Am J Med Genet A 2012; 158A 938-941.

25 Buckel T, Zaenglein AL: What syndrome is this? Ehlers-Danlos syndrome type VIII. Pediatr Dermatol 2007; 24: 189-191.

26 Li Y, Xu L, Hasturk H, Kantarci A, DePalma SR, Van Dyke TE: Localized aggressive periodontitis is linked to human chromosome 1q25. Hum Genet 2004, 114: 291-297.

27 Rahman N, Dunstan M, Teare MD et al: Ehlers-Danlos syndrome with severe early-onset periodontal disease (EDS-VIII) is a distinct, heterogeneous disorde with one predisposition gene at chromosome 12p13. Am J Hum Genet 2003; 73: 198-204.

28 Reinstein E, Wang RY, Zhan L, Rimoin DL, Wilcox WR: Ehlers-Danlos type VIII, periodontitis-type: further delineation of the syndrome in a four-generation pedigree. Am J Med Genet A 2011; 155: 742-747. 